\title{
Characterization of Fungal Morphology using Digital Image Analysis Techniques
}

Maria Papagianni*

Department of Hygiene and Technology of Food of Animal Origin, Faculty of Veterinary Medicine, Aristotle University of Thessaloniki, Greece

\begin{abstract}
The use of filamentous fungi for the production of commercially important products is old but keeps increasing during the last decades. New classes of compounds are being added in the list of products of fungal fermentations as a result of progress in methodologies and applications of biotechnology. Fungi are morphologically complex organisms that differ in structure throughout their life cycle. In submerged fermentation fungal morphology may take distinct forms ranging from dispersed filaments to densely interwoven masses of mycelium known as pellets. Each morphological form has each own characteristics that have a critical impact on the overall process outcome. Dispersed growth results in highly viscous broths with pseudoplastic behavior that have a negative impact on mass and energy transfer rates resulting in higher energy input requirements. Due to the high industrial relevance of fungal morphology there has been a substantial development of tools and techniques to characterize morphology and extract quantitative information that can be used in process control and optimization studies. Digital image analysis is the state of the art method to characterize and quantify fungal morphology in the developmental process from spores to filamentous structures to pellets. The progress made in the area since the 1990s, when the first image analysis methods were reported, is discussed in detail throughout the review.
\end{abstract}

Keywords: Digital image analysis; Fungal morphology; Fermentation

\section{Introduction}

Filamentous fungi are exploited in industry for the production of a wide range of compounds of commercial importance. Organic acids, antibiotics, enzymes, proteins (native and heterologous) and vitamins are produced efficiently by filamentous fungi in submerged fermentation systems in long-established processes [1]. Historically, the fermentation of these microorganisms was employed for the production of traditional oriental foods e.g. miso, tempeh, soy sauce and many others in solid state fermentation systems. This practice is still common in countries of the Far East while it attracts increasing industrial interest for the production of fungal metabolites due to simpler facilities and lower costs compared to those of submerged fermentation systems [2].

Filamentous fungi are morphologically diverse and complex organisms. Different morphological forms are found in submerged fermentations ranging from filamentous growth to compact pellets, with a whole array of morphological forms between the two extremes [3]. Their morphology at the sub-cellular level -the micro-morphology- also changes during the course of a submerged fermentation [3-5]. Factors affecting fungal morphology in submerged growth include the nature of the growth medium (presence of solids, type and concentration of the carbon source, levels of nitrogen and phosphate, trace minerals, $\mathrm{pH}$, dissolved oxygen and carbon dioxide), the type of the inoculum (vegetative or spores) and a large number of physical factors such as agitation, rheology, fermenter geometry, as well as the mode of culture itself e.g. batch, fed-batch or continuous culture [3]. It has been demonstrated in a large number of studies that fungal morphology has a strong effect on the productivity of mycelial processes and in many cases a certain morphological type has been associated with increased metabolite production [3]. From the large number of factors that affect morphology and their interrelationships, one can easily understand that control of fungal morphology in order to optimize production is a difficult task. It remains a very difficult task to extract quantitative relationships between process variables, metabolite production and fungal morphology in a fermentation process and therefore even more difficult to deduce general relationships between them. The role of many factors affecting fungal morphology is still not fully understood. However, and despite the difficulties, developing a thorough understanding of the role of process variables in morphological development and its subsequent impact on process productivities remains always a key target in the optimization of fungal fermentation processes.

To evaluate the importance of fungal morphology in a process, quantitative work is prerequisite. Great progress has been made in this area since the 1970s when investigations relied upon manual measurements performed under a microscope or on photographs were reported. Progress was made with the use of simple digitizing tables in the works of Metz et al. [6] and van Suijdam and Metz [7]. Progressively, with the availability of personal computers and digital video cameras, image analysis systems were developed. These were characterized by a low degree of automation [8] but soon highly automated systems were developed [9] with some degree of manual operation always needed for the production of accurate data. The later years have seen a substantial development of tools and techniques to characterize fungal morphology and extract quantitative information on morphological characteristics. Digital image analysis systems run with powerful software packages that permit fully automated work and statistical evaluation of the measured data $[10,11]$. Recently, confocal

*Corresponding author: Maria Papagianni, Department of Hygiene and Technology of Food of Animal Origin, School of Veterinary Medicine, Aristotle University of Thessaloniki, Thessaloniki 54124, Greece, Tel: +30 2310999804 ; Fax:+30 2310 999829; E-mail: mp2000@vet.auth.gr

Received April 07, 2014; Accepted April 25, 2014; Published April 30, 2014

Citation: Papagianni M (2014) Characterization of Fungal Morphology using Digital Image Analysis Techniques. J Microb Biochem Technol 6: 189-194. doi:10.4172/1948-5948.1000142

Copyright: ( 2014 Papagianni M. This is an open-access article distributed under the terms of the Creative Commons Attribution License, which permits unrestricted use, distribution, and reproduction in any medium, provided the original author and source are credited 
laser scanning microscopy (CLSM) and fluorescence microscopy have been employed successfully in fungal morphology studies offering precise structural analysis of the mycelium and spores [12-18].

This review highlights the progress made in the area of characterization of fungal morphology using image analysis techniques since the 1990s and presents selected relevant examples of applications.

\section{Digital Image Analysis Systems}

A typical image analysis system consists of the hardware and software components. The hardware components include the image source, which in the case of fungal morphology studies is a microscope, the image capture device, which is a monochrome or color video camera, a central processing unit (CCP), and the display monitor. Since the early 1990s when the first reports on digital image analysis of fungal morphology appeared, the progress in the area of relevant hardware has been enormous and will not be discussed in detail here. Once the image has been "frozen" and transferred to the computer, the relevant software is required to extract the data from it. Today, dedicated to image analysis software packages are available, ranging widely in abilities and costs. Their common characteristic in most cases is that they have been designed for application developers rather that for final users and the image analysis routines come as a library supported by a common programming language. The required sequence of operations can be encoded in the program to permit automatic repeatable analysis and measurements. The basic stages of the image analysis procedures are shown in Figure 1. Having completed the measurements and calculations, modern software packages offer the option the obtained data to be classified and further analyzed to give the final results in tabular or graphical form, and to be statistically treated making the need for use of statistical software mostly unnecessary.

Digital image analysis is the state of the art method to characterize and quantify fungal morphology in the differentiation process from spores to filamentous structures to pellets. Hyphal growth, septation and branching are commonly quantified in filamentous growth forms, while the number of features can be high enough in pelleted

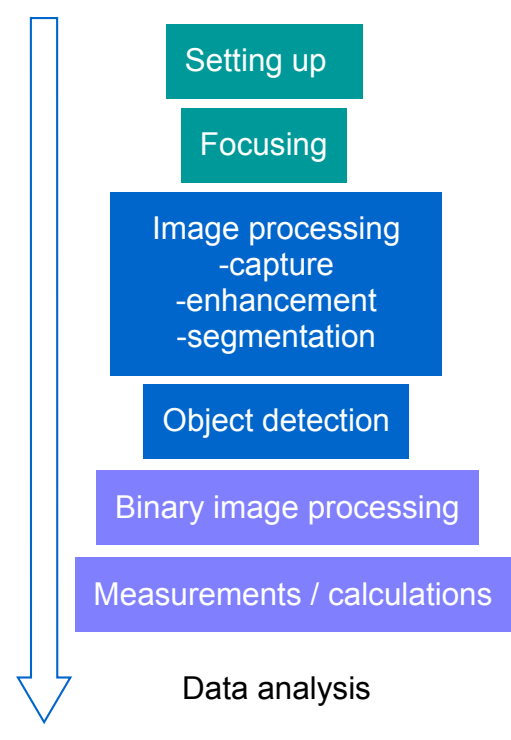

Figure 1: The basic steps of image analysis. morphologies to include, apart from the size-relevant, parameters that give a measure of shape such as circularity, compactness, roughness and convexivity.

\section{Characterization of Fungal Macro-Morphology}

\section{Filamentous structures}

The first significant advance in quantification of filamentous structures through image analysis was the work of Adams and Thomas [8] who described a semi-automatic system to perform measurements on filaments. The method was more accurate and faster than the digitizing tables used by Metz et al. [6] and van Suijdam and Metz [7] in the early 1980s although considerable manual intervention was still required, e.g. binary images were manually edited. In 1990, Packer and Thomas [19] presented a software application for use with a general purpose image analyzer that enabled fully automated analysis. The program required the manual setting of several application-specific parameters, e.g. grey- level and circularity thresholds, the specification of a "measuring frame" and a maximum length threshold. Hyphal elements were described in terms of the main hyphal length, number of tips, hyphal growth unit and other parameters, while for the first time the aggregated biomass -defined as "clumps"- was characterized and quantified. The method was tested on Streptomyces clavuligerus and Penicillium chrysogenum and compared to a manual image processing method (manual selection of mycelia and manual editing of binary images) proved to be only marginally faster due to the slow skeletonization algorithm - a problem that should have been overcome with the use of modern hardware.

Later, Tucker et al. [9] expanded the work of Packer and Thomas [19] by developing a method that could give important measurements on clumps. Clumps were identified by "ultimate skeletonization" which was successive removal of pixels until either a single point or a loop remained. Projected areas and perimeters of clumps were estimated through pixel counts and clumps were characterized in terms of their circularity and compactness. The method also permitted a detailed examination of mycelial structures beyond the classical hyphal growth unit. Having removed the small, "artificial" branches, free hyphal elements were subjected to a "shrink-back" algorithm that involved an iterative pruning of mycelia to identify branch-points and branches were classified as zero-order, first order etc. The method of Tucker et al. [9] was later used by Ammanulah et al. [20] to describe the dynamics of mycelial aggregation in batch and continuous cultures of Aspergillus oryzae.

The method of Tucker et al. [9] was further improved in the work of Paul and Thomas [21]. Mycelial trees, "simple" clumps and loose entanglements (clumps containing only 1-3 holes resulting from hyphal crossovers) were quantified in more detail and the morphology of $P$. chrysogenum in submerged culture was evaluated. A more detailed description of clumped morphology was proposed by Papagianni et al. [22] in fermentations of citric acid producing A. niger. Clumps were quantified in terms of their outer perimeter (P1), the perimeter of cores (P2) and the total length of filaments (l) that arose from the cores and their branches. The high degree of mycelial entanglement at the periphery of clumps made it impossible to distinguish between main filaments and branches and was suggested that $l$ could serve as an indication of the degree of branching. The hyphal diameter (d) was also determined. The image analysis method described by Papagianni et al. [22] involved significant manual intervention (mainly on the definition of the area of measurements) but a large number of steps 
Citation: Papagianni M (2014) Characterization of Fungal Morphology using Digital Image Analysis Techniques. J Microb Biochem Technol 6: 189194. doi:10.4172/1948-5948.1000142

in image processing and measurements were performed automatically. The method was later employed by the same group [23] to quantify the relationship between citric acid production and A. niger morphology as functions of the mixing intensity in a stirred tank and a tubular loop bioreactor and a set of empirical relationships were presented for the first time.

Figures 2 and 3 are photographs of filamentous mycelium of citric acid producer $A$. niger grown in submerged culture. Figure 3 shows a typical clump.

\section{Pelleted growth}

Pelleted morphologies (Figure 4) are common in submerged fungal fermentations. A number of image analysis methods have been proposed for the morphological characterization of fungal pellets. Reichl et al. [24] described the pellets of $S$. tendae by mean sizes, shape, content and frequency distributions. Pellet classification by means of the shape factor was introduced for the first time in that work. Cox and Thomas [25] proposed an image analysis method to characterize pellets based on the presence of a central core while they further classified them into smooth and "hairy" types. Pellet characterization using image analysis was reported by Durant et al. [26] who also described pellet zones using dyes, while later they improved their method using color image analysis [27].

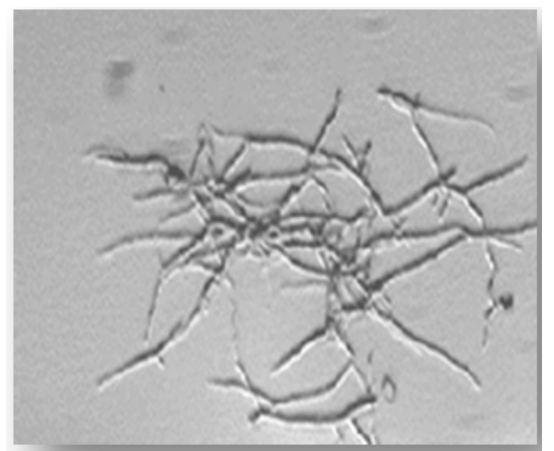

Figure 2: Filamentous growth (dispersed growth) of $A$. niger mycelium in submerged citric acid fermentation (x 200).

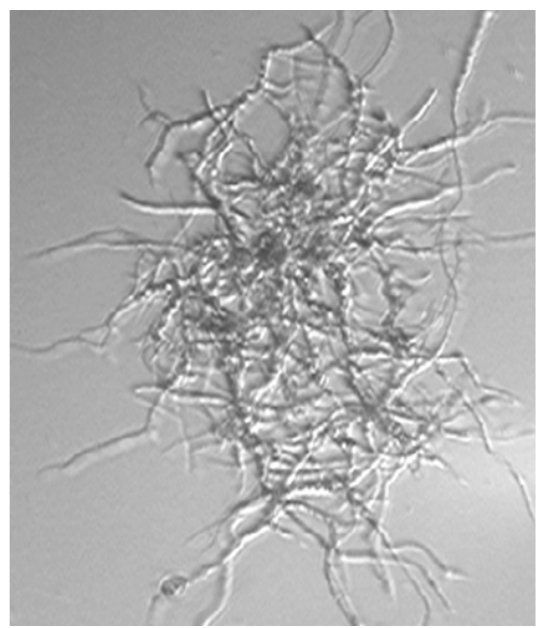

Figure 3: An A. niger clump in submerged citric acid fermentation (x 200).

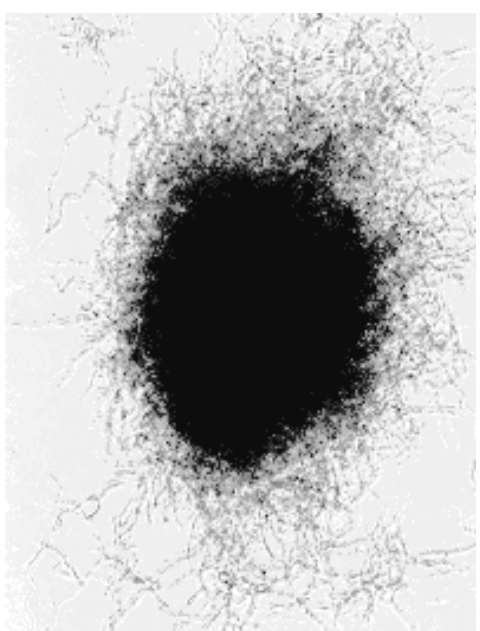

Figure 4: A typical fungal pellet by Aspergillus ficuum in submerged fermentation (x 100).

In the case of large pellets and pellets of the "hairy" type a combination of microscopic and macroscopic observations is often needed and different means of image capture are required. Paul and Thomas in their study with A. niger [21] suspended pellets in a cavity slide of $1 \mathrm{~mm}$ depth, suitable for mounting on the microscope's stage. For pellets of a larger that $0.6 \mathrm{~mm}$ diameter, a macro-viewer attached to a camera was required. A macro-viewer was used by Papagianni and Mattey [28] for the characterization of A. niger pellets in citric acid fermentation. O'Cleirigh et al. [29] in their work with S. hygroscopicus used a flatbed scanner to acquire images of safranin-stained pellets placed in Petri dishes in water suspension. The monochrome images were processed and binarized and the pellets were characterized in terms of their equivalent diameters, number and volume. Bizukojc and Ledakowicz [30] used a similar method with A. terreus pellets, although the Petri dishes were photographed rather than scanned. Rühl and Kües [31] placed the pellet suspension on a glass plate which was illuminated from below and acquired images which were further subjected to image analysis studies. It is obvious for the last studies that the set up work prior to image analysis was time consuming resulting in long overall image-processing times.

A number of studies have examined the filamentous region of pellets which vary in extent in various types of pellets e.g. smooth or "hairy" pellets. The filamentous fraction of pellets was separated from the core in the work of Paul and Thomas [21] at the step of the binary image processing. Similarly Park et al. [32], removed the pellet annular region in binary images by repeated opening cycles until only the pellet core remained. The filamentous area was calculated by subtracting the core from the total mycelial area. Also, Müller et al. [33] included in their work studies on the compactness of pellets of A. oryzae (estimated as the ratio of the projected area and the projected convex area of particles).

All morphological parameters mentioned above are presented in Table 1 along with their definitions [34].

\section{Characterization of fungal morphology at the level of the fine structure of hyphal elements}

The number of studies that focused on the fine structure of hyphal elements during fermentation remained very limited over 


\begin{tabular}{|c|c|}
\hline $\begin{array}{l}\text { Morphological } \\
\text { Parameters }\end{array}$ & Definition \\
\hline \multicolumn{2}{|l|}{ Measures of size } \\
\hline \multirow{3}{*}{$\begin{array}{l}\text { Area or projected area } \\
\text { (A) }\end{array}$} & $\begin{array}{l}\text { The area of projection of a 3-dimentional object into a 2-dimentional image. This is often represented by the sum of pixel values within the } \\
\text { region specified by the selected object and given by: }\end{array}$ \\
\hline & area $=\sum_{(i, j) \in A} \sum f i j$ \\
\hline & $\begin{array}{l}\text { A is the selected object, and }(\mathrm{i}, \mathrm{j}) \in \mathrm{A} \text {, all pixel locations within } \mathrm{A} \text {. The area is expressed as an actual area by multiplying by a calibration } \\
\text { constant squared. }\end{array}$ \\
\hline Distance & $\begin{array}{l}\text { Distances are line lengths in general, with the simplest of all distance measurements being that between two specified pixels. There are several } \\
\text { ways in which distances can be defined or approximated, as in the case of curved lines. }\end{array}$ \\
\hline Perimeter $(\mathrm{P})$ & Boundary lengths of objects. Since pixels lie on a rectilinear grid, it is necessary to include diagonal inter-pixel distances where appropriate. \\
\hline Convex perimeter $(\mathrm{Pc})$ & The length of the perimeter obtained by joining the outer points of an object, i.e. by filling in all the concavities in an object. \\
\hline Length (L) & $\begin{array}{l}\text { The length of a rectangular object having the same area and perimeter as the measured object. It is estimated from area and perimeter by } \\
L=\frac{P+\sqrt{p^{2}-16 \mathrm{~A}}}{4} \text {. } \\
\text { The maximum Feret diameter (Feret diameter }=\text { the diameter measured using a pair of calipers) is one definition of the Length of an object, } \\
\text { which may alternatively be specified as } \max _{(i, j),(k, l))_{A}} \sqrt{(i-k)^{2}+(j-l)^{2}}\end{array}$ \\
\hline Width (W) & $\begin{array}{l}\text { The width of a rectangular object having the same area and perimeter as the measured object. It is estimated from area and perimeter by } \\
W=\frac{P+\sqrt{p^{2}-16 \mathrm{~A}}}{4} \text {. } \\
\text { Alternatively it can be defined as the range of columns the object covers, i.e. } \max _{(i, j),(k, l) \in A}(j)-\operatorname{man}(j, j),(k, l) \in A \\
\text {, or as the Feret diameter at angle } \theta=0^{\circ} \text {. }\end{array}$ \\
\hline $\begin{array}{l}\text { Equivalent circular } \\
\text { diameter (D) }\end{array}$ & This is the diameter of a circle having the same area as the measured feature. It is estimated from area by $D=\sqrt{4 A / \pi}$. \\
\hline Height & This is the range of rows the object covers, i.e. $\underset{(i, j),(k . l) \in A}{\max (j)-\operatorname{man}_{(i, j),(k . l) \in A}(j)}$. It is the Feret diameter at angle $\theta=90^{\circ}$. \\
\hline \multicolumn{2}{|l|}{ Measures of shape } \\
\hline Circularity $(\mathrm{C})$ & $\begin{array}{l}\text { A shape factor that describes the deviation of an object in an image from a true circle. It is estimated from area and perimeter by } c=\frac{p^{2}}{4 \pi A} \text {. The } \\
\text { value of } 1 \text { corresponds to a circle, while larger values to shapes having a higher ratio of perimeter to area. }\end{array}$ \\
\hline $\begin{array}{l}\text { Compactness or } \\
\text { Fullness }(\mathrm{F})\end{array}$ & $\begin{array}{l}\text { Defined as the ratio of the area of an object to the area of a circle with the same perimeter. It is estimated by compactness }=4 \pi \frac{\text { area }}{(\text { perimeter })^{2}} \text {. It } \\
\text { is probably the most commonly used shape statistic and it is used to characterize the structure of mycelial clumps and pellets. A pellet without } \\
\text { hairy regions has } F \cong 1 \text { while a loose clump has } F<1 \text {. }\end{array}$ \\
\hline Roughness (R) & A measure of the irregularity of the perimeter of an object obtained from the circularity measurement around an object boundary. \\
\hline Convexivity & Convexivity can be obtained by forming the ratio of the perimeter of an object's hull to the perimeter of the object itself. \\
\hline \multicolumn{2}{|l|}{ Object count } \\
\hline Number of objects & Number of objects per field of view and cumulative counts for a sample. \\
\hline Hyphal tips & Number of tips per hypha, mean number of tips per hypha for a sample. \\
\hline
\end{tabular}

Table 1: The main morphological parameters of fungal morphology obtained through image analysis and their definitions according to Glasbey and Horgan [34].

the years. The structural complexity of hyphal elements of the filamentous growth form was evaluated for the first time by Paul et al. [35] using a fully automatic image analysis method. The method permitted the discrimination between cells filled with cytoplasmic material, vacuolated cells and degenerated, empty cells. Proportions of the classified objects were quantified. Their work was extended and presented in detail later with another couple of reports $[4,36]$. The methodology followed in these works involved a series of grey-scale and binary editing to extract vacuoles and present their distributions in volume and shape (circularity). The method was tested by monitoring the morphological parameters throughout fed-batch penicillin fermentation with $P$. chrysogenum. The extracted data was used for the construction of a powerful structured model for hyphal differentiation and penicillin production that was presented later by the same group [37].

In another work, Paul and Thomas [21] described an image analysis system designed to quantify vacuolation and active, growing regions of neutral red-stained $P$. chrysogenum mycelium. Through grey-scale and binary editing operations, valuoles and active hyphae were extracted from the image and measurements were followed. Their results showed that the vacuolated volume of hyphae increases during the course of a fed-batch fermentation and valuoles become larger and less circular while hyphal width (diameter) increased rapidly up to about 30 hours from inoculation to decline rapidly thereafter. These image analysis techniques permitted the quantified characterization of simple cellular differentiation of filamentous fungi and provide a valuable tool in studies of the relationship between differentiation and metabolite production.

A semi-automatic method for the characterization and quantification of vacuolation in citric acid producer $A$. niger fedbatch fermentations was presented by Papagianni et al. [5]. Images from phase-contrast microscopy (x 400) were processed and vacuoles were segmented through grey-level thresholding. Size and circularity filters were used to discriminate between vacuoles and various vacuole-like artifacts, and vacuoles were quantified automatically in terms of perimeter, diameter, circularity and area, while their volume was estimated assuming the hyphae to be cylindrical. Finally the percentage of vacuolated volume of filaments was calculated. The method was tested throughout citric acid fed-batch fermentations and time profiles of morphological parameters such as mean perimeter of 
clumps, length of filaments and vacuoles, along with kinetic parameters such as specific growth and production rates were used to establish a relationship between vacuolation, hyphal fragmentation and product formation under various agitation conditions and glucose levels. The results showed that vacuolation weakened the hyphae and low glucose levels created the conditions that favored fragmentation and made the mycelium susceptible to it when exposed to increased agitation conditions.

\section{Identification of the metabolically active fraction of the mycelium by image analysis studies}

In studies on the relationship between morphology and productivity, identification of the metabolically active fraction of the mycelium is often investigated. Fluorescence microscopy has proved a valuable tool for identifying active tips of filaments and active regions in pellets [38]. Fluorescent stains coupled with image analysis have been used in the works of Vanhoutte et al. [39], Agger et al. [40], Wongwicharn et al. [41], Hamanaka et al. [42] and Amanullah et al. [13]. Vanhoutte et al. [39] using a differential staining procedure and color image analysis in studies on the growth and differentiation of $P$. chrysogenum showed and quantified six physiological states: growing material (zone 1), three differentiated states characterized by increased granulation (zones 2,3,4), a highly vacuolated state (zone 5) and dead segments empty of cytoplasm (zone 6). Ager et al. [40] used a double staining procedure (calcofluor and DiOC6), fluorescence microscopy and automatic image analysis in studies on the morphology of $A$. oryzae. Organelles and cell walls were stained and the fraction of active cells within a hypha was determined using separate filter blocks to view the fluorescence produced from each stain. A morphologically structured model was constructed which was confirmed when applied to fed-batch and chemostat experiments. Amanullah et al. [13] used calcofluor white stain to distinguish between active and non-active tips in A. oryzae mycelium. Active tips appeared bright while inactive tips did not fluoresce. Their methodology involved a significant amount of manual work as the stained-active- tips had to be "cut" from the image. Calculations were mainly based on the grey-level threshold which was defined by the user and involved an amount of error since the point at which fluorescence was deemed may have varied. Also, tips within mycelia clumps could not be observed and therefore were not considered.

Fluorescent staining coupled with image analysis was also used to identify the active regions in pellets. Hamanaka et al. [42] studied the intracellular product distribution inside the pellets of arachidonic acid producer M. alpina. In another study, the same group used fluorescence microscopy and image analysis to localize lipid formation at the edge of M. alpina pellets [42]. Microtomed sections of pellets labeled with fluorescein isothiocyanate (FITC) and pellets stained with Nile red were subjected to image analysis using fluorescent microscopy and a cavity ratio was estimated on the average FITC intensity across the section diameter. FICT staining was found to be low at the center of pellets and particularly at the later stages of fermentation, indicating a hollow core (autolyzed mycelium) the size of which correlated with total pellet volume. Nile red was also restricted to the periphery of pellets giving the evidence that intracellular lipids were not present in the core of pellets. A similar methodology was followed by Bizukojc and Ledakowicz [30] to determine the active region in pellets of A. terreus. Microtome sections of methyl blue stained pellets were examined with fluorescent microscopy and their active peripheral region appeared reddish-violet while the interior greyish-white. The two regions were segmented and the volume of active biomass in each pellet was estimated based on the radius of the whole pellet and the radius of the inactive region.

Another work in which fluorescence microscopy was used along with image analysis is that by El-Enshasy et al. [14] who estimated the active fraction of biomass in pellets of A. niger. Acridine orange dye, indicative of active protein synthesis, was used to stain heat-fixed on microscope slides samples. The unproductive core of pellets appeared fluoresced green while the active periphery exhibited a strong redorange color. Images were analyzed manually in that work by drawing of diameters and estimating the depth of the active regions. Finally, the volume of productive mycelium was estimated in a similar way to that of Bizukojc and Ledakowicz [30].

\section{Conclusions}

Since the introduction of digital image analysis techniques, the amount of research carried out on the morphology of filamentous fungi has been impressive and therefore, the potential of image analysis has been well documented through these studies. The large efforts in the characterization of fungal morphology made evident the relationship between process parameters, fungal morphology and process productivities in a number of industrially important fermentations and facilitated the way for a further step in fungal biotechnology aiming at targeted engineering of fungal morphology. Quantitative information on mycelial differentiation has been used in the construction of structured models with predictive value and this understanding can be used in process optimization and design. The development of novel techniques in the area of morphological studies coupled with advances.

\section{References}

1. Arora DK (2004) Handbook of fungal biotechnology. Marcel Dekker, NY

2. Papagianni M (2014) Recent advances in solid-state fermentation applications for the food industry. Curr Biochem Eng 1: 2-8.

3. Papagianni M (2004) Fungal morphology and metabolite production in submerged mycelial processes. Biotechnol Adv 22: 189-259.

4. Paul GC, Kent CA, Thomas CR (1994) Hyphal vocuolation and fragmentation inpenicillium chrysogenum. Biotechnol Bioeng 44: 655-660.

5. Papagianni M, Mattey M, Kristiansen B (1999) Hyphal vacuolation and fragmentation in batch and fed-batch culture of Aspergillus niger and its relation to citric acid production. Process Biochem 35:359-366.

6. Metz B, de Bruijn EW, van Suijdam JC (1981) Method for quantitative representation of the morphology of molds. Biotechnol Bioeng 23: 149-162.

7. van Suijdam JC, Metz B (1981) Influence of engineering variables on the morphology of filamentous molds. Biotechnol Bioeng 23: 111-148.

8. Adams HL, Thomas CR (1988) The use of image analysis for morphological measurements on filamentous microorganisms. Biotechnol Bioeng 32: 707712 .

9. Tucker KG, Kelly T, Delgrazia P, Thomas CR (1992) Fully automatic measurement of mycelial morphology by image analysis. Biotechnol Progress 8: 353-359.

10. Barry D (2010) Development of novel image analysis methods for the morphological quantification of filamentous fungi. Ph.D. Thesis. School of Electrical Engineering Systems, Dublin Institute of Technology, Republic of Ireland.

11. Barry DJ, Williams GA (2010) Microscopic characterization of filamentous microbes: towards fully automated morphological quantification through image analysis. J Microsc 244: 1-20.

12. Krull R, Wucherpfennig T, Esfandabadi ME, Walisko R, Melzer G, et al. (2013) Characterization and control of fungal morphology for improved production performance in biotechnology. J Biotechnol 163: 112-123.

13. Amanullah A, Christensen LH, Hansen K, Nienow AW, Thomas CR (2002) 
Citation: Papagianni M (2014) Characterization of Fungal Morphology using Digital Image Analysis Techniques. J Microb Biochem Technol 6: 189194. doi: $10.4172 / 1948-5948.1000142$

Dependence of morphology on agitation intensity in fed-batch cultures of Aspergillus oryzae and its implications for recombinant protein production. Biotechnol Bioeng 77: 815-826.

14. El-Enshasy H, Kleine J, Rinas U (2006) Agitation effects on morphology and protein productive fractions of filamentous and pelleted growth forms of recombinant Aspergillus niger. Process Biochem 41: 2103-2112.

15. Herbrich S, Gehder M, Krull R, Gericke KH (2012) Label-free spatial analysis of free and enzyme-bound $\mathrm{NAD}(\mathrm{P}) \mathrm{H}$ in the presence of high concentrations of melanin. J Fluoresc 22: 349-355.

16. Smith AE, Moxham KE, Middelberg APJ (1998) On uniquely determining cellwall material properties with the compression experiment. Chem Eng Sci 53: 3913-3922.

17. Zhao L, Schaefer D, Marten MR (2005) Assessment of elasticity and topography of Aspergillus nidulans spores via atomic force microscopy. Appl Environ Microbiol 71: 955-960.

18. Fang TH, Kang SH, Hong ZH, Wu CD (2012) Elasticity and nanomechanical response of Aspergillus niger spores using atomic force microscopy. Micron 43: 407-411.

19. Packer HL, Thomas CR (1990) Morphological measurements on filamentous microorganisms by fully automatic image analysis. Biotechnol Bioeng 35: 870 881.

20. Amanullah A, Leonildi E, Nienow AW, Thomas CR (2001) Dynamics of mycelial aggregation in cultures of Aspergillus oryzae. Bioproc Biosyst Eng 24: 101-107.

21. Paul GC, Thomas CR (1998) Characterisation of mycelial morphology using image analysis. Adv Biochem Eng Biotechnol 60: 1-59.

22. Papagianni M, Mattey M, Kristiansen B (1994) Morphology and citric acid production of Aspergillus niger PM1. Biotechnol Lett 16: 929-934.

23. Papagianni M, Mattey M, Kristiansen B (1998) Citric acid production and morphology of Aspergillus niger as functions of the mixing intensity in a stirred tank and a tubular loop bioreactor. Biochem Eng J 2: 197-205.

24. Reichl U, King R, Gilles ED (1992) Characterization of pellet morphology during submerged growth of Streptomyces tendae by image analysis. Biotechnol Bioeng 39: 164-170.

25. Cox PW, Thomas CR (1992) Classification and measurement of fungal pellets by automated image analysis. Biotechnol Bioeng 39: 945-952.

26. Durant G, Cox PW, Formisyn P, Thomas CR (1994) Improved image analysis algorithm for the characterization of mycelial aggregates after staining. Biotechnol Techniques 8:759-764

27. Durant G, Crawley G, Formisyn P (1994) A simple staining procedure for the characterization of Basidiomyces pellets by image analysis. Biotechnol Techniques 8: 395-400.

28. Papagianni M, Mattey M (2004) Physiological aspects of free and immobilized
Aspergillus niger cultures producing citric acid under various glucose concentrations. Process Biochem 39: 1963-1970.

29. O'Cleirigh C, Walsh PK, O'Shea DG (2003) Morphological quantification of pellets in Streptomyces hygroscopicus var. geldanus fermentation broths using a flatbed scanner. Biotechnol Lett 25: 1677-1683.

30. Bizukojc M, Ledakowicz S (2010) The morphological and physiological evolution of Aspergillus terreus mycelium in the submerged culture and its relation to the formation of secondary metabolites. World J Microbiol Biotechnol 26: 41-54.

31. Rühl M, Kües U (2009) Automated image analysis to observe pellet morphology in liquid cultures of filamentous fungi such as the basidiomycete Coprinopsis cinerea. Curr Trends Biotechnol Pharmacy 3: 241-253.

32. Koike Y, Cai HJ, Higashiyama K, Fujikawa S, Park EY (2001) Effect of consumed carbon to nitrogen ratio of mycelial morphology and arachidonic acid production in cultures of Mortierella alpina. J Biosci Bioeng 91: 382-389.

33. Müller C, Hansen K, Szabo P, Nielsen J (2003) Effect of deletion of chitin synthase genes on mycelial morphology and culture viscosity in Aspergillus oryzae. Biotechnol Bioeng 81: 525-534.

34. Glasbey CA, Horgan GW (1994) Image analysis for the biological sciences. John Wiley \& Sons, Chichester, UK.

35. Paul GC, Kent CA, Thomas CR (1992) Quantitative characterization of vacuolization in Penicillium chrysogenum using automatic image analysis. Trans Inst Chem Eng 70: 13-20.

36. Paul GC, Kent CA, Thomas CR (1994) Image analysis for characterizing differentiation of Penicillium chrysogenum. Trans Inst Chem Eng 72: 95-105.

37. Paul GC, Thomas CR (1996) A structured model for hyphal differentiation and penicillin production using Penicillium chrysogenum. Biotechnol Bioeng 51 558-572.

38. Gull K, Trinci AP (1974) Detection of areas of wall differentiation in fungi using fluorescent staining. Arch Mikrobiol 96: 53-57.

39. Vanhoutte B, Pons MN, Thomas CR, Louvel L, Vivier H (1995) Characterization of Penicillium chrysogenum physiology in submerged cultures by color and monochrome image analysis. Biotechnol Bioeng 48: 1-11.

40. Agger T, Spohr AB, Carlsen M, Nielsen J (1998) Growth and product formation of Aspergillus oryzae during submerged cultivations: verification of a morphologically structured model using fluorescent probes. Biotechnol Bioeng 57: 321-329.

41. Wongwicharn A, McNeil B, Harvey LM (1999) Heterologous protein secretion and fungal morphology in chemostat cultures of a recombinant Aspergillus niger (B1-D). Enzyme Microb Technol 24: 489-497.

42. Hamanaka T, Higashiyama K, Fujikawa S, Park EY (2001) Mycelial pelle intrastructure and visualization of mycelia and intracellular lipid in a culture of Mortierella alpina. Appl Microbiol Biotechnol 56: 233-238. 